\title{
Active Contour Model Based on Local and Global Intensity Information for Medical Image Segmentation
}

\author{
Sanping Zhou ${ }^{\mathrm{a}}$, Jinjun Wang, ${ }^{\mathrm{a}, *}$, Shun Zhang ${ }^{\mathrm{a}}$, Yudong Liang ${ }^{\mathrm{a}}$, Yihong Gong ${ }^{\mathrm{a}}$ \\ ${ }^{a}$ The institute of artificial intelligence and robotic, Xi' an Jiaotong University, Xian Ning West Road \\ No.28, Shaanxi, 710049, P.R. China
}

\begin{abstract}
This paper proposes a novel region-based active contour model in the level set formulation for medical image segmentation. We define a unified fitting energy framework based on Gaussian probability distributions to obtain the maximum a posteriori probability (MAP) estimation. The energy term consists of a global energy term to characterize the fitting of global Gaussian distribution according to the intensities inside and outside the evolving curve, as well as a local energy term to characterize the fitting of local Gaussian distribution based on the local intensity information. In the resulting contour evolution that minimizes the associated energy, the global energy term accelerates the evolution of the evolving curve far away from the objects, while the local energy term guides the evolving curve near the objects to stop on the boundaries. In addition, a weighting function between the local energy term and the global energy term is proposed by using the local and global variances information, which enables the model to select the weights adaptively in segmenting images with intensity inhomogeneity. Extensive experiments on both synthetic and real medical images are provided to evaluate our method, show significant improvements on both efficiency and accuracy, as compared with the popular methods.
\end{abstract}

Keywords: Medical image segmentation, Intensity inhomogeneity, Level set method, Maximum a posteriori probability (MAP), Active contour model

\section{Introduction}

Image segmentation is a fundamental but important task in many image processing and computer vision applications, because the segmentation result will di-

\footnotetext{
*Corresponding author: Tel.: +86-029-83395146; fax: +86-029-83395175;

Email address: jinjun@mail.xjtu.edu.cn (Jinjun Wang)
} 
rectly affect the performance of the subsequent processing steps. A significant challenge of medical image segmentation is intensity inhomogeneity due to the bias field, which is caused by various factors, such as the spatial variations in illumination and the imperfection of imaging devices [1-3]. The intensity inhomogeneity can cause serious misclassifications among medical tissues, since the intensities in different tissue regions often overlap with each other. Therefore, how to deal with intensity inhomogeneity has been a challenging problem in medical image segmentation.

In the past decades, a number of researchers have been attracted to study the segmentation algorithm of images with intensity inhomogeneity and have proposed many effective methods [4-12]. The best known and most influential methods are active contour models, which have been widely used in image segmentation with promising results. The existing active contour models can be broadly categorized into two classes: edge-based models [13-18] and region-based models [19- -25].

The edge-based models utilize an edge descriptor as an additional constraint to stop the evolving curve on the object boundaries. Usually, a stopping function is used to attract the curves to the desired boundaries. For example, the GAC model [13] utilizes image gradient to construct an edge stopping function to stop the evolving curve on the object boundaries. Generally speaking, edge-based models have been successfully used for segmenting general images with strong object boundaries, but they are usually sensitive to noise and weak edges. Instead of utilizing image gradient as an edge descriptor, region-based models typically aim to identify each region of interest by using a certain region descriptor, such as intensity, color, texture or motion, to guide the motion of the evolving curve. Therefore, region-based models generally have better performance in segmenting images in the presence of noise and weak object boundaries [20]. In addition, region-based models are less sensitive to the initializations than edge-based models.

Among the region-based models, the piecewise constant (PC) model [19] is a representative one, which utilizes the global intensity information inside and outside of the evolving curve rather than the image gradient driving the motion of the evolving curve. Similarly, Cremers et al. [20] adopted the global intensity mean and variance information to define a global Gaussian distribution fitting (GGDF) energy to guide the evolving curve to stopping on the object boundaries. However, both of them fail to segment images with intensity inhomogeneity because they assume that the intensities in each region maintain a constant, which is inaccurate in images with intensity inhomogeneity. Later in 2001 and 2002, Tsai et al. [21] and Vese et al. [22] independently proposed two similar region-based models, widely known as piecewise smooth (PS) models, for segmenting more general images. Although the PS model does not assume the homogeneity of image intensities, they can get a desirable result in segmenting images with intensity inhomogeneity. 
However, the computation of the PS model are too expensive.

Recently, local intensity information has been incorporated into the active contour models to deal with images with intensity inhomogeneity. For example, Li et al. [23, 24] proposed a local binary fitting (LBF) model to overcome the difficulty in segmentation caused by intensity inhomogeneity. The LBF model utilizes local intensity information and thus is able to deal with intensity inhomogeneity. However, the LBF model can't distinguish regions with different intensity variances because it only uses local mean information to guide the motion of the evolving curve. Therefore, more complete statistical characteristics of local intensity have to be taken into account. For instance, Rosenhahn et al. [25] takes advantage of both local intensity mean and variance information to characterize the local intensity distribution in their proposed active contour model. However, the local intensity mean and variance are defined empirically in their model. In [4], Wang et al. proposed a local Gaussian distribution fitting (LGDF) model in the level set formulation with local intensity mean and variance information, in which the local mean and variance are strictly derived from a variational principle instead of being defined empirically. As a result, the LGDF model can not only deal images with intensity inhomogeneity, but also can distinguish regions with different intensity variances. However, the LGDF model assumes the a prior probability that describes a pixel belonging to the partition of a neighborhood to be a constant, which leads to inaccurate results in segmenting small objects. What's more, it is sensitive to initializations, for it only uses the local intensity information to drive the motion of the evolving curve.

In this paper, we present a novel region-based active contour model based on local and global intensity information in the level set formulation for medical image segmentation. The main advantages of our method can be highlighted as follows:

(1) Both global and local intensity information are incorporated into the proposed segmentation framework based on the MAP rule. In the resulting energy functional, the global energy term accelerates the evolution of the evolving curve far away from the object's boundaries, while the local energy term guides the evolving curve near the object's boundaries to stop on them. As a result, both efficiency and accuracy of segmentation can be improved under the proposed segmentation framework.

(2) In order to segment the small details in the image, the a priori probability that describes a pixel belonging to the partition of a neighborhood is modeled by using the size information of partition at two sides of the evolving curve. Compare with the LGDF model, in which the a priori probability was assumed to be a constant, our method can get higher accuracy, especially in segmenting the small details. 
(3) A weighting function between the global energy term and the local energy term is proposed by using the local and global variance information as a good feature to characterize the distance between the evolving curve and the object boundary. As a result, the weights can be selected adaptively in the process of segmenting medical images.

The rest of the paper is organized as follows: Some related models are reviewed in Section 2. The proposed method is introduced briefly in Section 3 . Detailed simulation results are provided in Section 4. Concluding remarks are given in Section 5 .

\section{Background models}

\subsection{The GGDF model}

Cremers et al. [20] proposed a global Gaussian distribution fitting (GGDF) model based on MAP rule to segment the image domain $\Omega$ into $N$ disjointed regions $\left\{\Omega_{i}\right\}_{i=1}^{N}$ in the level set formulation, where $\Omega=\bigcup_{i=1}^{N} \Omega_{i}, \forall \Omega_{i} \cap \Omega_{j}=$ $\phi, i \neq j$. Let $p\left(x \in \Omega_{i} \mid I(x)\right)$ be the a posteriori probability of region $\Omega_{i}$ given the gray value $I(x)$, the MAP estimation of image segmentation could be expressed by the Bayes rule as follow:

$$
p\left(x \in \Omega_{i} \mid I(x)\right)=\frac{p\left(I(x) \mid x \in \Omega_{i}\right) p\left(x \in \Omega_{i}\right)}{p(I(x))},
$$

where $p\left(I(x) \mid x \in \Omega_{i}\right)$, denoted by $p_{i}(I(x))$, is the probability in region $\Omega_{i}$ and $p\left(x \in \Omega_{i}\right)$ is the a posteriori probability of the region $\Omega_{i}$. Because the priori probability $p(I(x))$ of gray value $I(x)$ is independent of the choice of the region and can therefore be neglected for simplicity in image segmentation.

Given the priori a probability $p\left(x \in \Omega_{i}\right)=1 / N$, therefore the term $p\left(x \in \Omega_{i}\right)$ can be ignored. Assuming that the pixels within each region are independent, the MAP will be achieved only if $p_{i}(I(x))$ across the image domain $\Omega$ is maximized: $\prod_{i=1}^{N} \prod_{x \in \Omega_{i}} p_{i}(I(x))$. Taking a logarithm, the maximization is converted into minimizing the following energy:

$$
E^{G G D F}=\sum_{i=1}^{N} \int_{\Omega_{i}}-\log p_{i}(I(x)) d x .
$$

Assuming the probability density $p_{i}(I(x))$ to be Gaussian with mean $\mu_{i}$ and standard variance $\sigma_{i}$ in region $\Omega_{i}, p_{i}(I(x))$ can be formulated as follows:

$$
p_{i}(I(x))=\frac{1}{\sqrt{2 \pi} \sigma_{i}} \exp \left(-\frac{\left(\mu_{i}-I(x)\right)^{2}}{2 \sigma_{i}^{2}}\right) .
$$


By introducing the level set function $\phi(x)$ to represent the regions $\left\{\Omega_{i}\right\}_{i=1}^{N}$, Eq. (2) can be rewritten as:

$$
E^{G G D F}=\sum_{i=1}^{N} \int_{\Omega}-\log p_{i, x}(I(x)) M_{i}(\phi(x)) d x .
$$

where $M_{i}(\phi)$ is the characteristic function of region $\Omega_{i}$, which satisfies $\sum_{i=1}^{N} M_{i}=$ $1, M_{i} M_{j}=0, \forall i \neq j$.

In the GGDF model, both the intensity mean $\mu_{i}$ and intensity variance $\sigma_{i}$ are introduced to fit the image intensities inside and outside of the evolving curve, respectively. If the images are distorted by intensity inhomogeneity, pixel values from different regions will overlap with each other, which makes the global fitting framework inaccurate [26]. This is the reason why the GGDF model cannot segment medical images in presence of intensity inhomogeneity.

\subsection{The LGDF model}

In [4], Wang et al. proposed a local Gaussian distribution fitting (LGDF) model in the level set formulation to segment images with intensity inhomogeneity. Let $O_{x}=\{y:|x-y| \leq \rho\}$ be a circular neighborhood with radius $\rho$ centered at point $x$. The $N$ disjointed regions $\left\{\Omega_{i}\right\}_{i=1}^{N}$ induce a partition of the neighborhood $O_{x}$ which can be denoted by $\left\{\Omega_{i} \cap O_{x}\right\}_{i=1}^{N}$. The MAP estimation of image segmentation in the circular neighborhood can be expressed using the Bayes rule as follows:

$$
p\left(y \in \Omega_{i} \cap O_{x} \mid I(y)\right)=\frac{p\left(I(y) \mid y \in \Omega_{i} \cap O_{x}\right) p\left(y \in \Omega_{i} \cap O_{x}\right)}{p(I(y))},
$$

where $p\left(y \in \Omega_{i} \cap O_{x} \mid I(y)\right)$ is the a posteriori probability of the partition $\Omega_{i} \cap O_{x}$ given the intensity value $I(y)$ in the circle neighborhood $O_{x}, p\left(y \in \Omega_{i} \cap \mathrm{O}_{x}\right)$ is the a priori probability of the partition $\Omega_{i} \cap \mathrm{O}_{x} \cdot p\left(I(y) \mid y \in \Omega_{i} \cap O_{x}\right)$, denoted by $p_{i, x}(I(y))$, is the probability density in the partition $\Omega_{i} \cap \mathrm{O}_{x}$, and $p(I(y))$ is the a priori probability of gray value $I(y)$, which is independent of the choice of the region and can therefore be neglected.

Given the a priori probability $p\left(y \in \Omega_{i} \cap O_{x}\right)=1 / N$, therefore the term $p\left(y \in \Omega_{i} \cap \mathrm{O}_{x}\right)$ can be ignored. Assuming that the pixels within each region are independent, the MAP will be achieved only if the product of $p_{i, x}(I(y))$ across the neighborhood $O_{x}$ is maximized: $\prod_{i=1}^{N} \prod_{y \in \Omega_{i} \cap O_{x}} p_{i, x}(I(y))$. Taking a logarithm, the maximization can be converted into minimizing the following energy:

$$
E_{x}^{L G D F}=\sum_{i=1}^{N} \int_{\Omega_{i} \cap O_{x}}-\log p_{i, x}(I(y)) d y,
$$


The probability density $p_{i, x}(I(y))$ in Eq. (6) is modeled by Gaussian distribution with mean $\mu_{i}$ and variance $\sigma_{i}$ in partition $\Omega_{i} \cap O_{x}$. Specifically,

$$
p_{i, x}(I(y))=\frac{1}{\sqrt{2 \pi} \sigma_{i}(x)} \exp \left(-\frac{\left(\mu_{i}(x)-I(y)\right)^{2}}{2 \sigma_{i}^{2}(x)}\right) .
$$

By introducing a non-negative weighting function which satisfies $\omega(x-y)=0$ for $|x-y| \geq \rho$ and $\int_{O_{x}} \omega(x-y) d y=1$ into Eq. (6), and using the level set function $\phi(y)$ to represent the partitions $\left\{\Omega_{i} \cap O_{x}\right\}_{i=1}^{N}$, the energy functional in Eq. (6) can be rewritten as follows:

$$
E_{x}^{L G D F}=\sum_{i=1}^{N} \int_{\Omega_{i}}-\omega(x-y) \log p_{i, x}(I(y)) M_{i}(\phi(y)) d y,
$$

where $M_{i}(\phi)$ is the characteristic function of partition $\Omega_{i} \cap O_{x}$, which satisfies $\sum_{i=1}^{N} M_{i}=1, M_{i} M_{j}=0, \forall i \neq j$.

Now the overall energy $E^{L G D F}$ with all points $x$ in the image domain $\Omega$ can be formulated as:

$$
E^{L G D F}=\int\left(\sum_{i=1}^{N} \int_{\Omega_{i}}-\omega(x-y) \log p_{i, x}(I(y)) M_{i}(\phi(y)) d y\right) d x .
$$

Since the LGDF model utilizes the local intensity mean $\mu_{i}$ and the local intensity variance $\sigma_{i}$ simultaneously, it is more robust to images with intensity inhomogeneity and can segment regions with different intensity variances well. The limitation with the LGDF model is due to the a priori probability $p\left(y \in \Omega_{i} \cap O_{x}\right)$ which is assumed to be a constant during the evolution. This is the reason why the LGDF model cannot segment small details well. Besides, the LGDF model is very sensitive to the initializations, since it only utilizes the local intensity information to drive the evolving curve.

\section{The proposed model}

Motivated by the GGDF model and the LGDF model, in this section, we present our model that consists of three parts: the global energy term $E^{G}$, the local energy term $E^{L}$, and the regularization energy term $E^{R}$. The overall energy is formulated as:

$$
E=g E^{L}+(1-g) E^{G}+E^{R}
$$

where $g$ is the weighting function between the global energy term $E^{G}$ and the local energy term $E^{L}$. 


\subsection{The energy terms}

The global energy term $E^{G}$ is directly derived from Eq. (4) in the GGDF model, which is defined based on the global properties by using the intensity mean and the intensity variance inside and outside of the evolving curve $C$, respectively. Therefore, the global energy term $E^{G}$ is defined as follows:

$$
E^{G}=\sum_{i=1}^{N} \int_{\Omega}-\log p_{i, x}(I(x)) M_{i}(\phi(x)) d x .
$$

where $M_{i}(\phi)$ is the characteristic function of region $\Omega_{i}$, which satisfies $\sum_{i=1}^{N} M_{i}=$ $1, M_{i} M_{j}=0, \forall i \neq j$.

Due to the use of the global intensity mean $\mu_{i}$ and the global intensity variance $\sigma_{i}$ in the global energy term $E^{G}$, the evolution of the evolving curve $C$ can be accelerated towards the object's boundaries. However, for the medical images with intensity inhomogeneity, the final obtained curve $C$ can hardly divide the image into object region and background region even after a long iteration time. The reason is that the global energy term assumes that both the intensity mean and the intensity variance are piecewise constant. As a result, the constant intensity mean and variance cannot represent the inhomogeneous intensities in both the object region and the background region. In order to achieve a good performance in segmenting medical images with intensity inhomogeneity, the local information has to be taken into account.

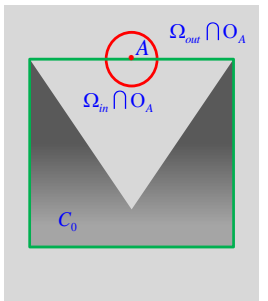

(a)

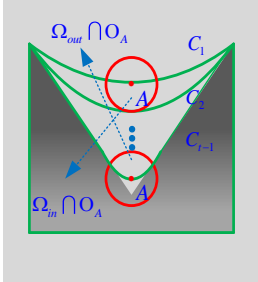

(b)

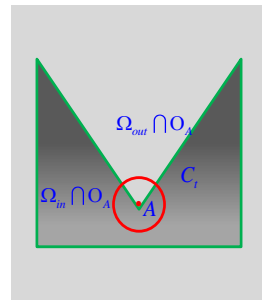

(c)

Figure 1: Changes of the partitions $\Omega_{i} \cap O_{A}$ during the curve evolution. Green curve denotes the evolving curve and read curve denotes the circular neighborhood. (a) shows initialization of the evolving curve, (b)shows the changes of the partitions during the evolution, (c) shows the final state of the partitions after $t$ iterations

In the LGDF model, the a priori probability $p\left(y \in \Omega_{i} \cap O_{x}\right)$ is directly defined as a constant, which is unreasonable because the size of the partition $\Omega_{i} \cap O_{x}$ changes during the whole evolution process. As shown in Fig. 1, considering a circle neighborhood centered at point $A$, the size of partition $\Omega_{\text {out }} \cap O_{A}$ decreases as the curve $C$ evolves to the object boundary, while the size of partition $\Omega_{i n} \cap O_{A}$ 
increases simultaneously. Let $S\left(\Omega_{i} \cap O_{x}\right)$ and $S\left(O_{x}\right)$ denote the size of partition $\Omega_{i} \cap O_{x}$ and the neighborhood $O_{x}$, respectively. Therefore, we can define a function with respect to the size of partition $\Omega_{i} \cap O_{x}$, such as $p\left(y \in \Omega_{i} \cap O_{x}\right)=S\left(\Omega_{i} \cap\right.$ $\left.O_{x}\right) / S\left(O_{x}\right)$, to model the a priori probability $p\left(y \in \Omega_{i} \cap O_{x}\right)$ more reasonable. By incorporating the a prior probability function in Eq. (9), the local energy term $E^{L}$ can then be defined as follows:

$E^{L}=\sum_{i=1}^{N} \iint_{\Omega}-\omega(x-y)\left(\log p_{i, x}(I(y))+\log \left(\frac{S\left(\Omega_{i} \cap O_{x}\right)}{S\left(O_{x}\right)}\right)\right) M(\phi(y)) d x d y$,

where $\omega(x-y)$ is a non-negative weighting function. In this paper, we define $\omega(x-y)$ to be a truncated Gaussian kernel by:

$$
\omega(x-y)=\left\{\begin{array}{cc}
\frac{1}{\sqrt{2 \pi} \sigma} \exp \left(-\frac{|x-y|^{2}}{2 \sigma^{2}}\right) & ,|x-y| \leq \rho \\
0 & , \text { otherwise }
\end{array} .\right.
$$

where $\sigma$ is the standard deviation of the Gaussian function, and $\rho$ is the radius of the circular neighborhood $O_{x}$.

In order to choose the weight between the global energy term $E^{G}$ and the local energy term $E^{L}$ adaptively, we propose a weighting function based on the observation as shown in Fig. 1. At the beginning of evolution, the curve $C$ divide the image domain into one homogeneous region and one inhomogeneous region, while the circle neighborhood will remain inside the background region, as shown in Fig. 1 a). When the evolving curve $C$ approaches the object's boundary, the circle neighborhood is constituted of one homogeneous part and one inhomogeneous part, as shown in Fig. 1 (b). By the time the evolving curve $C$ stops at the object boundary, the image domain is divide into two homogeneous regions and the circle neighborhood is constituted of two homogeneous parts, as shown in Fig. 11(c). Since the intensity variation is small in the homogeneous region and large in the inhomogeneous region, the difference between $\sigma_{i}(x)$ and $\sigma_{i}$ provides a good feature to characterize the distance between the evolving curve and the object boundary. Therefore, the weighting function is defined as follows:

$$
g=\frac{1}{Z} \exp \left(-\int_{\Omega}\left(\sigma_{i n}(x)-\sigma_{i n}\right)^{2} M_{i}(\phi) d x\right) .
$$

where $Z$ is a normalization factor, $\sigma_{i n}$ is the intensity variance inside the evolving curve and $\sigma_{i n}(x)$ is the intensity variance inside the evolving curve in the circle neighborhood. 
For more accurate computation involving the level set function and its evolution, we need to regularize the level set function by penalizing its deviation from a signed distance function [15], which can be defined by the following energy function:

$$
P(\phi)=\int_{\Omega} \frac{1}{2}(|\nabla \phi-1|)^{2} d x,
$$

As in typical level set methods, we often need to regularize the zero level set by penalizing its length to derive a smooth contour during the evolution. In this paper, it is formulated as follows:

$$
L(\phi)=\int_{\Omega}|\nabla H(\phi)| d x=\int_{\Omega} \delta(\phi)|\nabla \phi| d x,
$$

where $H(x)$ is the Heaviside function, and $\delta(x)$ is the Dirac function.

So, in the proposed model, the regularization term $E^{R}$ should be composed of two terms, which can be formulated as follows:

$$
E^{R}=\mu L(\phi)+v P(\phi)=\mu \int_{\Omega} \delta(\phi)|\nabla \phi| d x+v \int_{\Omega} \frac{1}{2}(|\nabla \phi-1|)^{2} d x .
$$

where $\mu, v$ are the coefficients of the length penalty term and the distance penalty term, respectively.

\subsection{The level set formulation}

We first consider the case of $N=2$. In this case, the image domain $\Omega$ is partitioned into two regions $\left\{\Omega_{i}\right\}_{i=1}^{2}$, which can be represented by $\Omega_{1}=\{\phi>0\}$ and $\Omega_{2}=\{\phi<0\}$. In this circumstance, the proposed model in Eq. [10] is defined by:

$$
\begin{aligned}
& E\left(\phi, u_{i}(x), \sigma_{i}(x), u_{i}, \sigma_{i}\right)=\mu \int_{\Omega} \delta_{\varepsilon}(\phi)|\nabla \phi| d x+\nu \frac{1}{2} \int_{\Omega}(|\nabla \phi|-1)^{2} d x \\
& +g \sum_{i=1}^{2} \iint_{\Omega}-\omega(x-y)\left(\log p_{i, x}(I(y))+\log \left(\frac{S\left(\Omega_{i} \cap O_{x}\right)}{S\left(O_{x}\right)}\right) M_{i}(\phi) d x d y\right. \\
& +(1-g) \sum_{i=1}^{2} \int_{\Omega}-\log p_{i, x} I(x) M_{i}(\phi) d x .
\end{aligned}
$$

where $M_{1}(\phi)=H_{\varepsilon}(\phi)$ and $M_{2}(\phi)=1-H_{\varepsilon}(\phi) . H_{\varepsilon}(x)$ is the non-compactly supported, smooth and strictly monotone approximation of $H(x)$, and $\delta_{\varepsilon}(x)$ is the regularized approximation of $\delta(x)$, which are defined as follows:

$$
H_{\varepsilon}(x)=\frac{1}{\pi}\left[1+\frac{2}{\pi} \arctan \left(\frac{x}{\varepsilon}\right)\right], \delta_{\varepsilon}(x)=\frac{1}{\pi} \cdot \frac{\varepsilon}{\varepsilon^{2}+x^{2}} .
$$


where $\varepsilon$ is a positive constant, we choose $\varepsilon=1$.

Calculus of variations would show that the functions $u_{i}(x), \sigma_{i}(x)$ and constants $u_{i}, \sigma_{i}$ that minimize the energy functional $E\left(\phi, u_{i}(x), \sigma_{i}(x), u_{i}, \sigma_{i}\right)$ in Eq. (18) for a fixed function $\phi$ can be given by:

$$
\begin{gathered}
u_{i}(x)=\frac{\int_{\Omega} \omega(x-y) I(y) M_{i}(\phi) d y}{\int_{\Omega} \omega(x-y) M_{i}(\phi) d y} . \\
\sigma_{i}^{2}(x)=\frac{\int_{\Omega} \omega(x-y)\left(u_{i}(x)-I(y)\right)^{2} M_{i}(\phi) d y}{\int_{\Omega} \omega(x-y) M_{i}(\phi) d y} . \\
u_{i}=\frac{\int_{\Omega} I(x) M_{i}(\phi) d x}{\int_{\Omega} M_{i}(\phi) d x} . \\
\sigma_{i}^{2}=\frac{\int_{\Omega}\left(u_{i}-I(x)\right)^{2} M_{i}(\phi) d x}{\int_{\Omega} M_{i}(\phi) d x} .
\end{gathered}
$$

The energy functional $E\left(\phi, u_{i}(x), \sigma_{i}(x), u_{i}, \sigma_{i}\right)$ in Eq. (18) with respect to $\phi$ can be minimized using the gradient descent flow equations:

$$
\begin{aligned}
& \frac{\partial \phi}{\partial t}=\mu \delta_{\varepsilon}(\phi) \operatorname{div}\left(\frac{\nabla \phi}{|\nabla \phi|}\right)+\nu\left(\Delta \phi-\operatorname{div}\left(\frac{\nabla \phi}{|\nabla \phi|}\right)\right)-\delta_{\varepsilon}(\phi) \log \frac{p_{1}(I(x))}{p_{2}(I(x))} \\
& -\delta_{\varepsilon}(\phi) \int_{\Omega} \omega(x-y) \log \left(\frac{p_{1}(I(y)) \int_{\Omega} \omega(x-y) M_{1}(-\phi) d y}{p_{2}(I(y)) \int_{\Omega} \omega(x-y) M_{2}(-\phi) d y}\right) d y .
\end{aligned}
$$

Next to extend the method to segment the image domain $\Omega$ into $N=2^{n}$ regions, multiple level set functions $\Phi=\left\{\phi_{i}\right\}_{i=1}^{n}$ could be used to represent the regions $\left\{\Omega_{i}\right\}_{i=1}^{N}$. For example, two level set functions would divide the image domain into $N=4$ regions. Therefore, the proposed model in Eq. (10) can be formulated as:

$$
\begin{aligned}
& E\left(\Phi, u_{i}(x), \sigma_{i}(x), u_{i}, \sigma_{i}\right)=(1-g) \sum_{i=1}^{4} \int_{\Omega}-\left(\log p_{i, x} I(x) M_{i}(\Phi)\right) d x \\
& +g \sum_{i=1}^{4} \iint_{\Omega}-\omega(x-y)\left(\log p_{i, x}(I(y))+\log \frac{S\left(\Omega_{i} \cap O_{x}\right)}{S\left(O_{x}\right)}\right) M_{i}(\Phi) d x d y \\
& +\mu \sum_{i=1}^{2} \int_{\Omega} \delta_{\varepsilon}\left(\phi_{i}\right)\left|\nabla \phi_{i}\right| d x+\nu \sum_{i=1}^{2} \frac{1}{2} \int_{\Omega}\left(\left|\nabla \phi_{i}\right|-1\right)^{2} d x .
\end{aligned}
$$

Functions $u_{i}(x), \sigma_{i}(x)$ and constants $u_{i}, \sigma_{i}$ that minimize the energy functional $E\left(\Phi, u_{i}(x), \sigma_{i}(x), u_{i}, \sigma_{i}\right)$ for a fixed $\Phi$ can be given by:

$$
u_{i}(x)=\frac{\int_{\Omega} \omega(x-y) I(y) M_{i}(\Phi) d y}{\int_{\Omega} \omega(x-y) M_{i}(\Phi) d y} .
$$




$$
\begin{gathered}
\sigma_{i}^{2}(x)=\frac{\int_{\Omega} \omega(x-y)\left(u_{i}(x)-I(y)\right)^{2} M_{i}(\Phi) d y}{\int_{\Omega} \omega(x-y) M_{i}(\Phi) d y} . \\
u_{i}=\frac{\int_{\Omega} I(x) M_{i}(\Phi) d x}{\int_{\Omega} M_{i}(\Phi) d x} . \\
\sigma_{i}^{2}=\frac{\int_{\Omega}\left(u_{i}-I(x)\right)^{2} M_{i}(\Phi) d x}{\int_{\Omega} M_{i}(\Phi) d x} .
\end{gathered}
$$

where $M_{i}(\Phi)$ is the regional characteristic function, which can be formulated as follows:

$$
M_{i}(\Phi)=\prod_{j=1}^{2}\left(1-b_{i-1}^{j}\right)+(-1)^{1+b_{i-1}^{j}} H_{\varepsilon}\left(\phi_{j}\right) .
$$

where $b_{i-1}^{1}, b_{i-1}^{2}$ is the binary representation of $i-1$.

Similar to Eq. (18), minimizing the energy functional $E\left(\Phi, u_{i}(x), \sigma_{i}(x), u_{i}, \sigma_{i}\right)$ in Eq. (25) with respect to $\Phi$ can be achieved by solving the following gradient descent flow equations:

$$
\begin{aligned}
\frac{\partial \phi_{1}}{\partial t}= & -\delta_{\varepsilon}\left(\phi_{1}\right)\left(H_{\varepsilon}\left(\phi_{2}\right)\left(e_{4}-e_{2}\right)+\left(1-H_{\varepsilon}\left(\phi_{2}\right)\right)\left(e_{3}-e_{1}\right)\right) \\
& +\mu \delta_{\varepsilon}\left(\phi_{1}\right) \operatorname{div}\left(\frac{\nabla \phi_{1}}{\left|\nabla \phi_{1}\right|}\right)+\nu\left(\Delta \phi_{1}-\operatorname{div}\left(\frac{\nabla \phi_{1}}{\left|\nabla \phi_{1}\right|}\right)\right), \\
\frac{\partial \phi_{2}}{\partial t}= & -\delta_{\varepsilon}\left(\phi_{2}\right)\left(H_{\varepsilon}\left(\phi_{1}\right)\left(e_{4}-e_{2}\right)+\left(1-H_{\varepsilon}\left(\phi_{1}\right)\right)\left(e_{3}-e_{1}\right)\right) \\
& +\mu \delta_{\varepsilon}\left(\phi_{2}\right) \operatorname{div}\left(\frac{\nabla \phi_{2}}{\left|\nabla \phi_{2}\right|}\right)+\nu\left(\Delta \phi_{2}-\operatorname{div}\left(\frac{\nabla \phi_{2}}{\left|\nabla \phi_{2}\right|}\right)\right) .
\end{aligned}
$$

where $e_{i}$ is defined as follows:

$$
\left.e_{i}=\int_{\Omega} \omega(x-y) \log \left(p_{i}(I y)\right) \int_{\Omega} \omega(x-y) M_{i}(-\phi) d y\right) d x+\log p_{i}(I(x)) .
$$

\subsection{Implementation and evaluation}

The partial derivatives in Eq. 24, Eq. (31) and Eq. (32) can be solved by a finite difference method in numerical scheme. All the partial derivatives can be approximated by the central difference, and the temporal partial derivatives can be approximated by the forward difference. The level set functions can be simply initialized as a binary step function, which takes a negative constant value $\phi_{0}=-2$ (or $\Phi_{0}=-2$ )inside the evolving curve and a positive constant value $\phi_{0}=2$ (or $\Phi_{0}=2$ ) outside it. To compute the convolutions in Eq. (20), Eq. 21), Eq. 26, , 
Eq. (27) and Eq. (33) more efficiently, the kernel $\omega$ is truncated as a $(2 \rho+1) \times$ $(2 \rho+1)$ mask, where $\rho$ is no less than $2 \sigma$.

In summary, the implementation of our proposed method is revisited in Algorithm 1, where $L\left(C_{i}\right)$ represents the length of the evolving curve at the $i-t h$ iteration, and $\eta$ is a constant which is set to be 0.001 in the following experiments.

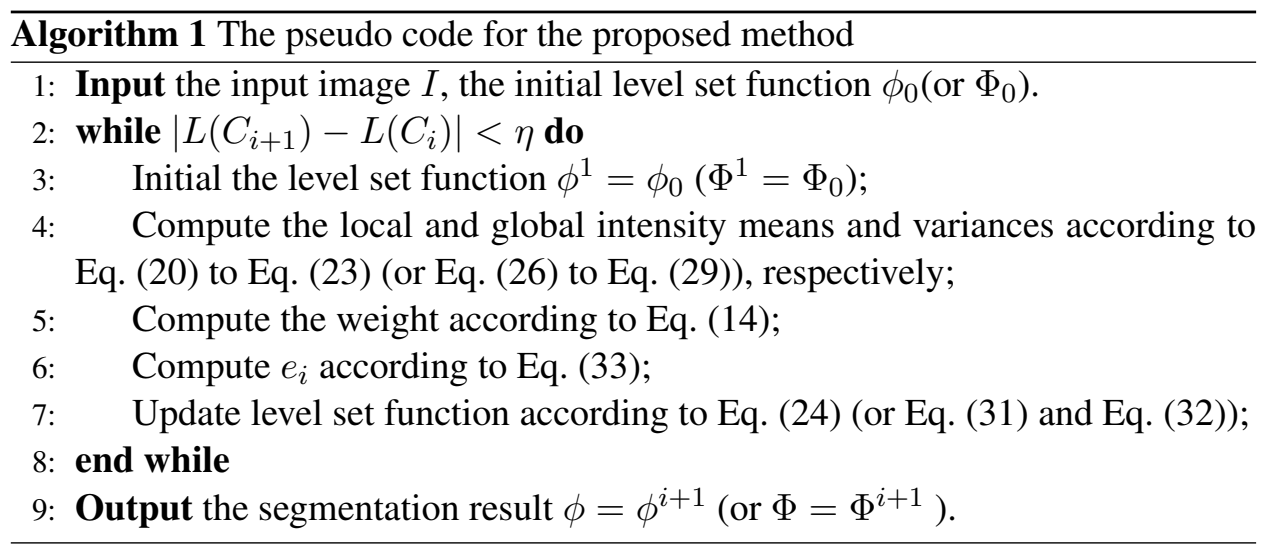

For the quantitative comparisons with the respective works, commonly used validation metrics, such as the true positive (TP), the false positive (FP), the precision (P), and the Jaccard similarity (JS), are used to evaluate the segmentation performance. The definitions and descriptions of these validation metrics are listed in Table 11, where $S_{G}$ is ground truth region, $S_{T}$ is the segmented region by the computerized test method, $\uparrow$ indicates that higher scores are better and $\downarrow$ means the opposite. What's more, the CPU time and iterations consumed in each experiment are used to compare the efficiency with the other methods.

Table 1: The definitions and descriptions of validation metrics

\begin{tabular}{|c|c|c|}
\hline Name & Definition & Description \\
\hline True positive $(\uparrow)$ & $T P=\frac{\left|S_{G} \cap S_{T}\right|}{S_{G}}$ & $\begin{array}{l}\text { TP denotes the percentage of the postive pixels that both } \\
\text { in the segmented region and the ground truth region. }\end{array}$ \\
\hline False positive $(\downarrow)$ & $F P=\frac{\left|S_{G} \cup S_{T}-S_{G}\right|}{S_{G}}$ & $\begin{array}{l}\text { FP denotes the percentage of the postive pixels that in } \\
\text { the segmented region but not in the ground truth region. }\end{array}$ \\
\hline Precision $(\uparrow)$ & $P=\frac{T P}{T P+F P}$ & $\begin{array}{l}\mathrm{P} \text { denotes the proportion of the postive pixels that have } \\
\text { been rightly segmented in the segmented region. }\end{array}$ \\
\hline Jaccard similarity $(\uparrow)$ & $J S=\frac{\left|S_{G} \cap S_{T}\right|}{\left|S_{G} \cup S_{T}\right|}$ & $\begin{array}{l}\text { JS is a statistical measure of similarity between the } \\
\text { ground truth region and the segmented region. }\end{array}$ \\
\hline
\end{tabular}




\section{Experimental results}

This section presents experimental results on both synthetic and real medical images. The proposed model was implemented by MATLAB 7.0 on a computer with Intel Core 2 Duo 2.2GHz CPU, 2G RAM, and Windows 7 operating system. Unless otherwise specified, we use the following parameters throughout the experiments: time step $\Delta t=0.1$, parameter of Gaussian kernel $\sigma=3.0$, radius of the circle neighborhood $\rho=6$, coefficient of length penalty energy term $\sigma=0.0001 \times 255^{2}$, coefficient of distance penalty energy term $u=1$.

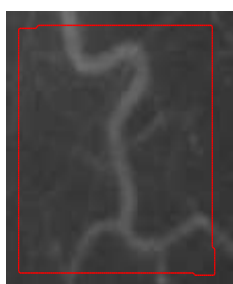

(a)

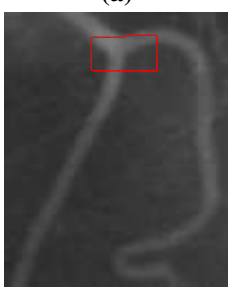

(f)

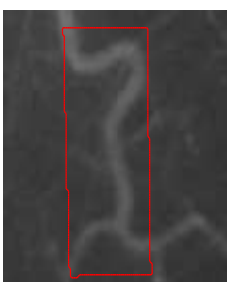

(b)

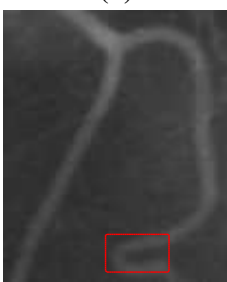

(g)

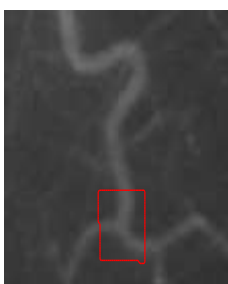

(c)

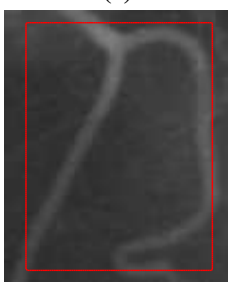

(h)

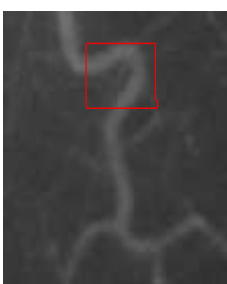

(d)

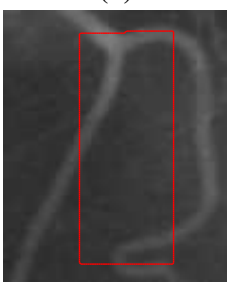

(i)

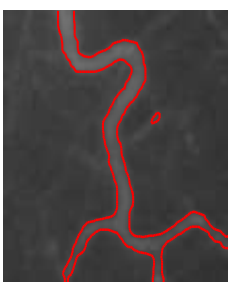

(e)

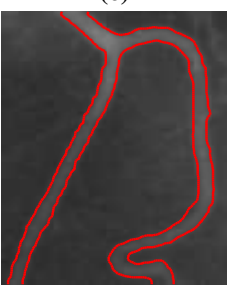

(j)

Figure 2: Robustness of our method to different initializations. (a)-(d) and (f)-(i) show four different initializations to the two blood vessel images, respectively. (e) and (j) show the final segmentation results of the proposed method under these initializations.

In the first experiment, we show the results of our method in segmenting two real blood vessel images, as shown in Fig. 2, to demonstrate its robustness under different initializations. Fig. 2(a)-(d) and Fig. 2(f)-(i) show four different initializations of the level set function, respectively. Fig. 2(e) and Fig. 2(j) show the corresponding segmentation results of our method in segmenting the two real blood vessel images under the four different initializations, respectively. It can be seen that no matter where the initial curve was placed in the image, our method can reach it's global minimum in the proposed circumstances. In order to show how the adaptive weight changes during the whole segmentation, we record the value of the weighting function at each iteration in segmenting the two real blood vessel images, as shown in Fig. 3. It can be clearly seen that the weights increase gradually with the evolving curve reaching the object boundary, which leads to the global 
energy term plays the major role at the beginning while the local energy term plays the major role at the end.

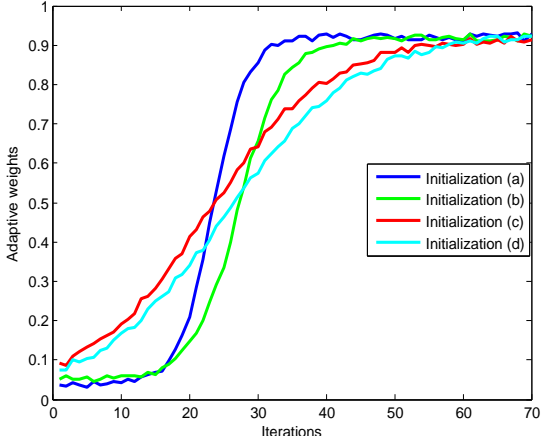

(a)

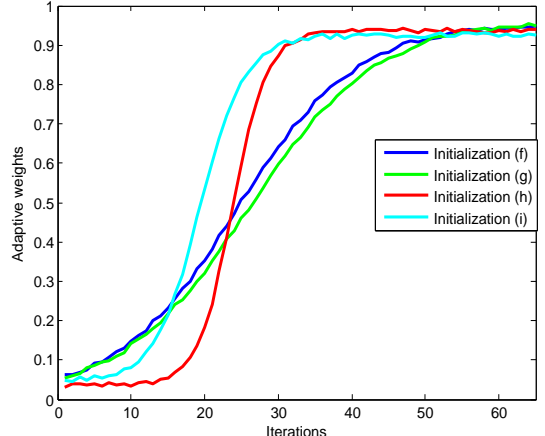

(b)

Figure 3: The adaptive weight changes in segmenting the two real blood vessel images in Fig. 2 under four different initializations. (a) shows the weights' change in segmenting the image in the first row, and (b) shows the weights change' in segmenting the image in the second row.

Table 2: Accuracy influence of the Gaussian kernel and radius of circle neighborhood to our method

\begin{tabular}{clllll}
\hline \multirow{2}{*}{ Accuracy metric } & \multicolumn{5}{c}{$\sigma=2.0$} \\
\cline { 2 - 6 } & $\rho=4$ & $\rho=5$ & $\rho=6$ & $\rho=7$ & $\rho=8$ \\
\hline TP(AVG \pm STD $)$ & $0.8174 \pm 0.0100$ & $0.8392 \pm 0.0095$ & $0.8517 \pm 0.0006$ & $0.8642 \pm 0.0136$ & $0.8714 \pm 0.0155$ \\
FP(AVG \pm STD) & $0.0186 \pm 0.0233$ & $0.0125 \pm 0.0151$ & $0.0098 \pm 0.0113$ & $0.0129 \pm 0.0151$ & $0.0144 \pm 0.0170$ \\
$\mathrm{P}(A V G \pm$ STD) & $0.9783 \pm 0.0270$ & $0.9855 \pm 0.0173$ & $0.9887 \pm 0.0130$ & $0.9853 \pm 0.0172$ & $0.9837 \pm 0.0192$ \\
JS(AVG \pm STD) & $0.8239 \pm 0.0078$ & $0.8323 \pm 0.0127$ & $0.8477 \pm 0.0076$ & $0.8656 \pm 0.0075$ & $0.8688 \pm 0.0048$ \\
\hline Accuracy Metric & & \multicolumn{5}{c}{$\sigma=3.0$} \\
\hline TP(AVG \pm STD) & $0.8263 \pm 0.0059$ & $0.8904 \pm 0.0563$ & $0.9918 \pm 0.0004$ & $0.9920 \pm 0.0016$ & $0.9918 \pm 0.0008$ \\
FP(AVG \pm STD) & $0.0095 \pm 0.0113$ & $0.0098 \pm 0.0120$ & $0.0121 \pm 0.0144$ & $0.0107 \pm 0.0122$ & $0.0112 \pm 0.0126$ \\
$\mathrm{P}($ AVG \pm STD) & $0.9888 \pm 0.0133$ & $0.9989 \pm 0.0126$ & $0.9880 \pm 0.0142$ & $0.9894 \pm 0.0121$ & $0.9889 \pm 0.0124$ \\
JS(AVG \pm STD) & $0.8395 \pm 0.0074$ & $0.8692 \pm 0.0041$ & $0.9138 \pm 0.0035$ & $0.9138 \pm 0.0023$ & $0.9178 \pm 0.0038$ \\
\hline
\end{tabular}

In the second experiment, we show how different values of the Gaussian kernel and radius of the circle neighborhood affect the performance of our method. The two initializations and manual segmentation results of a synthetic image and a CT image are shown in the first column of Fig. 4. In the first two sets of experiments, we specify the value of the Gaussian kernel $\sigma=3.0$ and change the radius of the circle neighborhood from $\rho=4.0$ to $\rho=8.0$, to demonstrate the influence of the radius of the circle neighborhood to our method. In order to demonstrate the influence of the values of Gaussian kernel to our method, we specify the value of the Gaussian kernel $\sigma=2.0$ and change the radius of the circle neighborhood from $\rho=4.0$ to $\rho=8.0$ in the last two sets of experiments. The segmentation results of 
$\rho=4.0$ to $\rho=8.0$ are shown in the second column to the sixth column in Fig. 4 . respectively. In order to compare the influence of different values of Gaussian kernel and radius of the circle neighborhood to our method quantitatively, we give the accuracy evaluation and the efficiency evaluation in each set of experiment in Table 2 and Fig. 5, respectively. From the results, we can conclude that our method can get its better performance in both accuracy and efficiency by setting the radius $\sigma=3.0$ and the Gaussian kernel $\rho=6.0$.

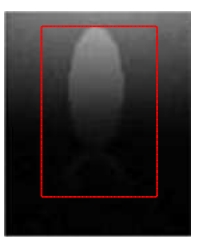

(a)

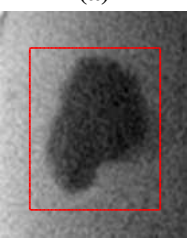

(g)

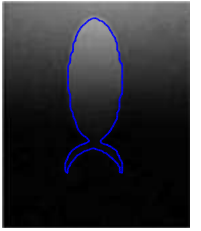

(m)

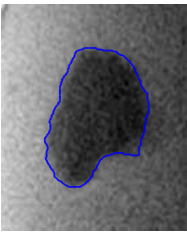

(s)

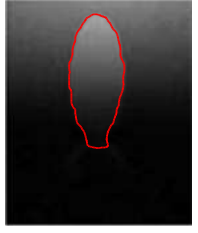

(b)

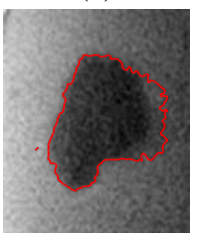

(h)

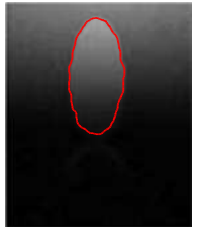

(n)

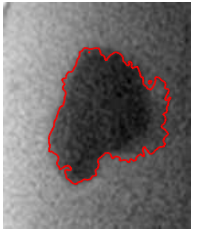

(t)

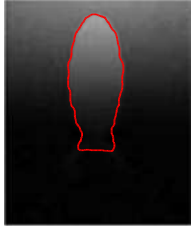

(c)

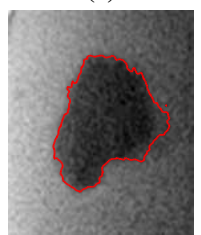

(i)

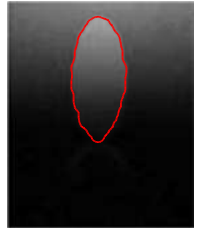

(o)

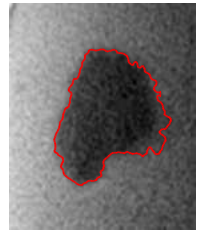

(u)

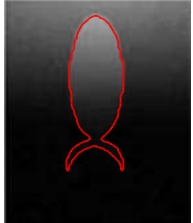

(d)

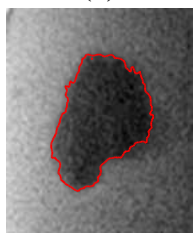

(j)

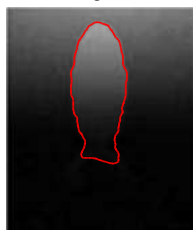

(p)

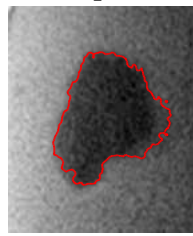

(v)

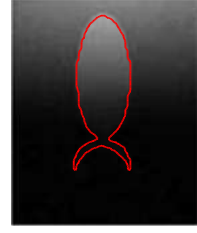

(e)

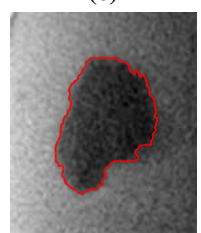

(k)

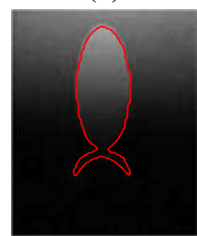

(q)

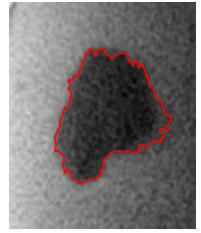

(w)

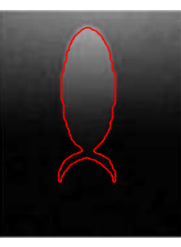

(f)

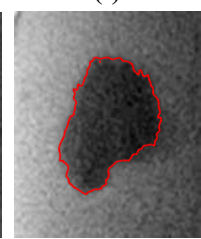

(I)

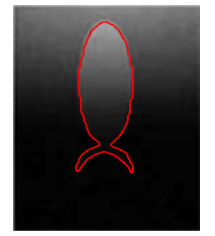

(r)

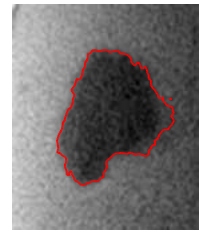

(x)

Figure 4: Influence of different values of the Gaussian kernel and radius of the circle neighborhood to our method. The first column shows two initializations and manual segmentation results, the second row and the third row show the segmentation results with $\sigma=3.0$, the fourth row and the fifth row show the segmentation results with $\sigma=2.0$. The second column to the sixth column show the segmentation results of $\rho=4.0$ to $\rho=8.0$, respectively.

In the third experiment, we compare our method with some well known method in segmenting three kinds of synthetic images, as shown in Fig.6, to illustrate the performance of these methods in segmenting the images with intensity inhomo- 


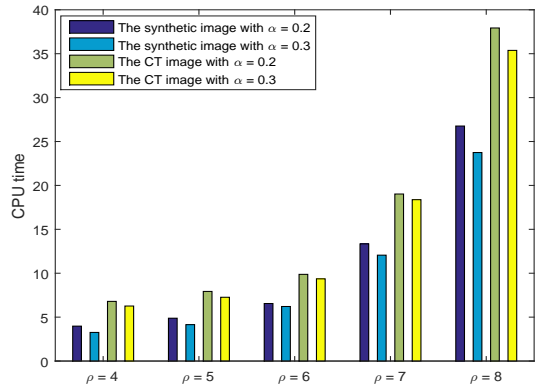

(a)

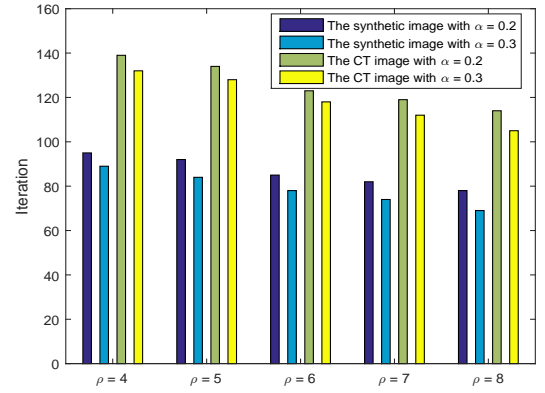

(b)

Figure 5: Efficiency influence of the Gaussian kernel and radius of circle neighborhood to our method. (a) and (b) show the CPU time and iterations in segmenting the two images shown in Fig. 4 respectively.

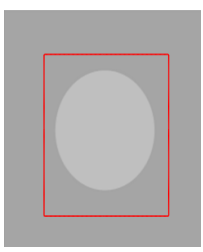

(a)

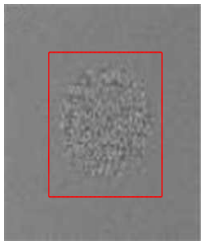

(g)

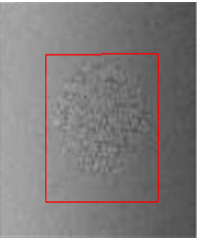

(m)

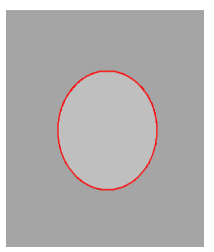

(b)

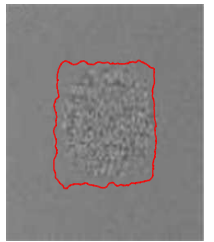

(h)

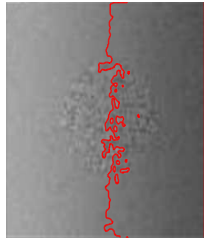

(n)

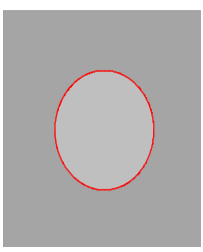

(c)

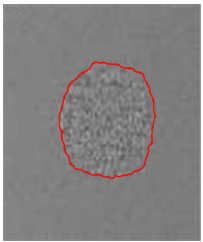

(i)

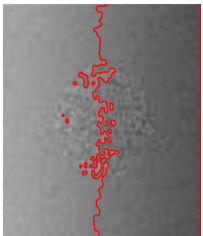

(o)

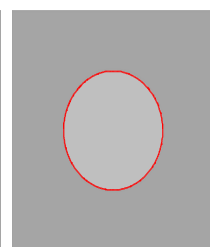

(d)

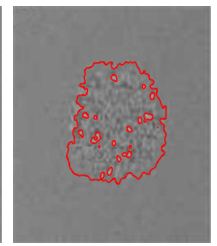

(j)

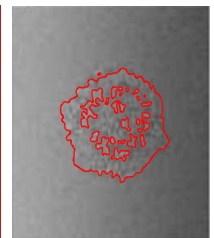

(p)

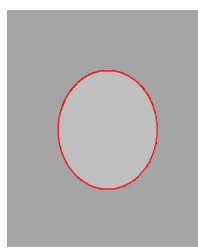

(e)

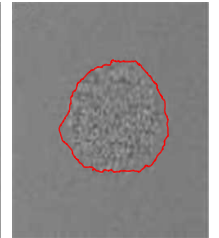

(k)

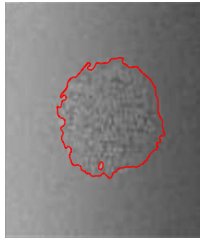

(q)

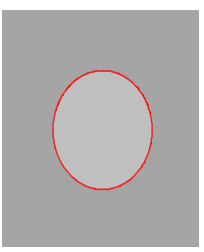

(f)

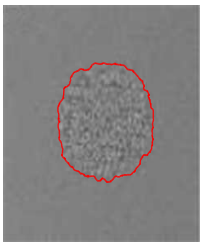

(I)

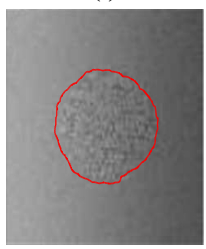

(r)

Figure 6: Comparison with some well known methods in segmenting a synthetic image. The first column shows three different initializations, and the second to the sixth columns show the segmentation results of CV model, GGDF model, LBF model, LGDF model and our method, respectively.

geneity and varying intensity variance. The first column shows three similar initializations to the three kinds of images, namely the binary image, the image with varying intensity variance, and the image with both varying intensity variance and 
intensity inhomogeneity. The second column to the sixth column show the segmentation results of the CV model, the GGDF model, the LBF model, the LGDF model and our method, respectively. From the segmentation results shown in Fig, 6 , we can see that all the five methods can segment the binary image well, while the CV model, the GGDF model and the LBF model cannot get desirable results in segmenting the last two kinds of images. Thanks to the local mean and variance information used in the LGDF model and our method, the two methods can do well in segmenting the three kinds of image.

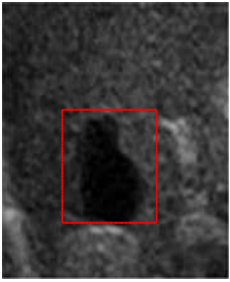

(a)

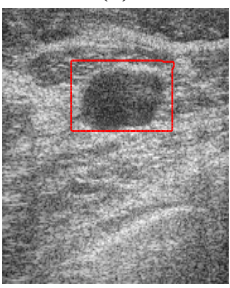

(f)

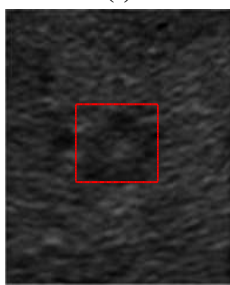

(k)

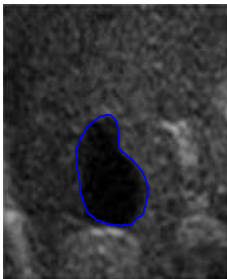

(b)

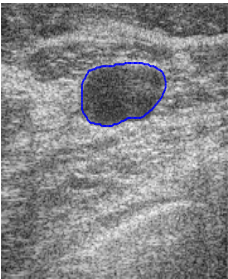

(g)

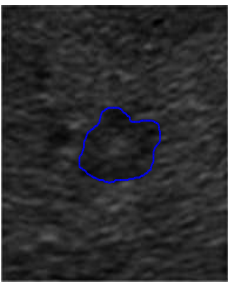

(1)

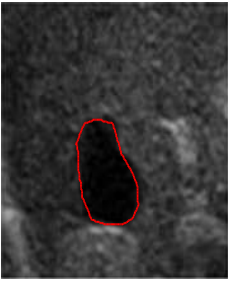

(c)

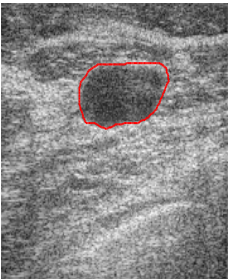

(h)

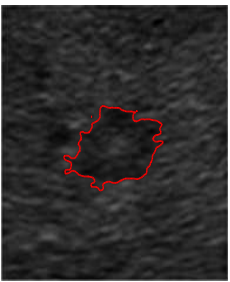

(m)

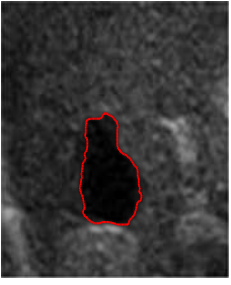

(d)

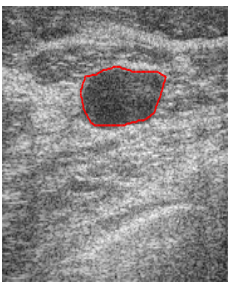

(i)

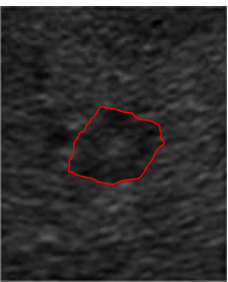

(n)

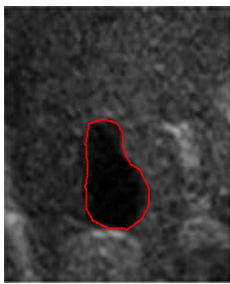

(e)

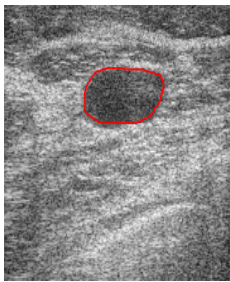

(j)

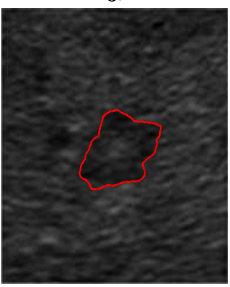

(o)

Figure 7: Comparison with some well known local and global method in segmenting three ultrasound images. The first column shows three different initializations, the second column show the manual segmentation results, and the third to the fifth columns show the segmentation results of LCV model, LGIF model and our method respectively.

In the fourth experiment, we compare our method with the LCV model and the LGIF model in segmenting three ultrasound images. The segmentation results are shown in Fig.7, where the first column shows three different initializations, the second column shows the manual segmentation results, and the third column to the fifth column show the segmentation results of the LCV model, the LGIF model 
and our method, respectively. In order to compare the three methods quantitatively, we give the accuracy measure and efficiency measure of each method in segmenting the three ultrasound images in Table 3 and Table 4, respectively. The three methods can do well in segmenting the three ultrasound images, and our method perform better than the other two methods in both accuracy and efficiency because of the adaptive weighting function and the the priori probability function are used simultaneously in the proposed method.

Table 3: Accuracy metrics obtained by different local and global methods

\begin{tabular}{cllll}
\hline \multirow{2}{*}{ Method } & \multicolumn{4}{c}{ Performance metric } \\
\cline { 2 - 5 } & $\mathrm{TP}(\mathrm{AVG} \pm \mathrm{STD})$ & $\mathrm{FP}(\mathrm{AVG} \pm \mathrm{STD})$ & $\mathrm{P}(\mathrm{AVG} \pm \mathrm{STD})$ & $\mathrm{JS}(\mathrm{AVG} \pm \mathrm{STD})$ \\
\hline LCV & $0.9550 \pm 0.0093$ & $0.0394 \pm 0.0047$ & $0.9609 \pm 0.0058$ & $0.8748 \pm 0.0070$ \\
LGIF & $0.9701 \pm 0.0153$ & $0.0349 \pm 0.0039$ & $0.9718 \pm 0.0042$ & $0.8815 \pm 0.0105$ \\
Our method & $0.9780 \pm 0.0183$ & $0.0287 \pm 0.0039$ & $0.9718 \pm 0.0042$ & $0.8990 \pm 0.0153$ \\
\hline
\end{tabular}

Table 4: Efficiency metrics obtained by different local and global methods

\begin{tabular}{cccccccc}
\hline \multirow{2}{*}{ Image } & \multirow{2}{*}{ Size } & \multicolumn{2}{c}{ LCV model } & \multicolumn{2}{c}{ LGIF model } & \multicolumn{2}{c}{ Our method } \\
\cline { 3 - 7 } & & CPU time & Iteration & CPU time & Iteration & CPU time & Iteration \\
\hline Fig.1 & $180 \times 190$ & 12.5392 & 157 & 11.8299 & 145 & 8.6743 & 109 \\
Fig.2 & $180 \times 180$ & 11.8932 & 147 & 10.9625 & 136 & 8.2304 & 103 \\
Fig.3 & $194 \times 194$ & 12.9527 & 164 & 12.2331 & 154 & 9.3256 & 121 \\
\hline
\end{tabular}

In the fifth experiment, we compared our method with the LBF model, the LGDF model, the LCV model and the LGIF model in segmenting three real brain MR images. The segmentation results are shown in Fig. 8, where the first column shows the three different initializations, and the second column to the sixth column show the corresponding results of the five methods respectively. From the segmentation results shown in Fig. 8(b) to Fig. 8.e) and Fig. 8(m) to Fig. 8(q), we can see that all of the LBF model, the LGDF model, the LCV model and the LGIF model can't segment the small details out in the bottom of the two images, while our method can segment all the small details well. From the segmentation results shown in the second row, we can see that all of the LBF model, the LGDF model, the LCV model and the LGIF model fails to segment the small details in the left of the image, while our method can segment all the small details well. The superior performance by our method is due to the fact that we use the size information at the two sides of the evolving curve in local region to model the priori probability, in this way, we are able to tell whether a pixel belongs to the partition of a neighborhood instead of assuming it to be a constant. 


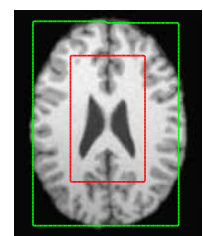

(a)

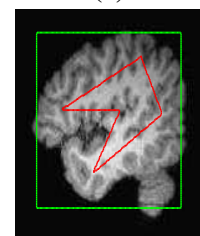

(g)

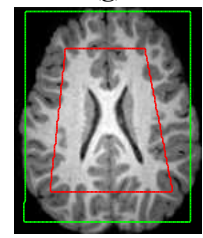

(m)

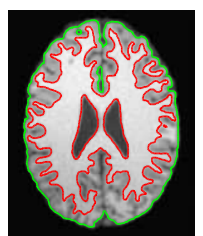

(b)

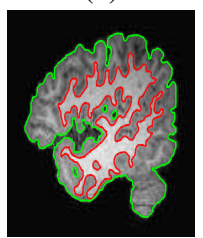

(h)

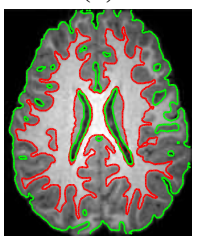

(n)

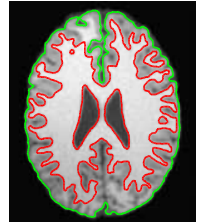

(c)

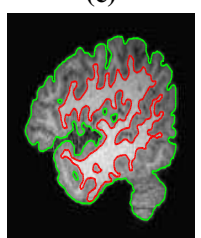

(i)

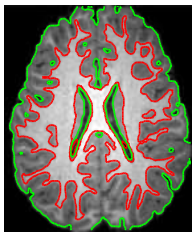

(o)

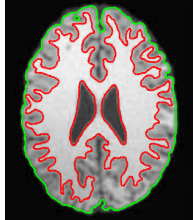

(d)

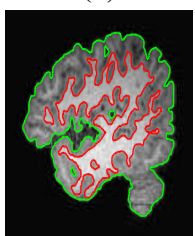

(j)

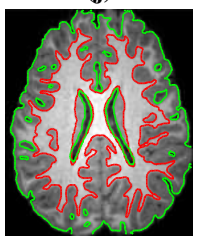

(p)

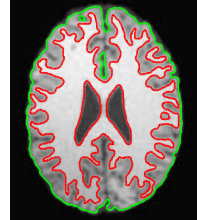

(e)

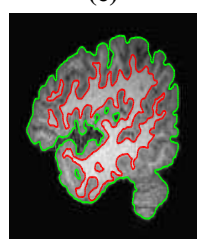

(k)

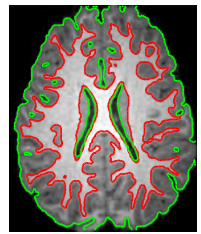

(q)

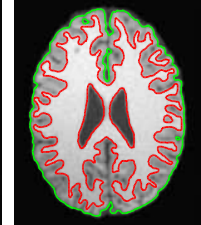

(f)

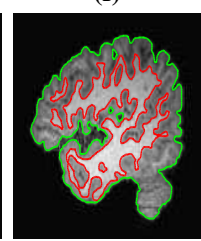

(I)

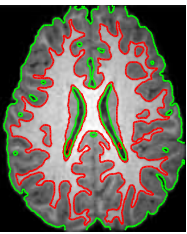

(r)

Figure 8: Comparison with some well known methods in segmenting three real brain MR images. The first column shows three different initializations; the second column to the sixth column show the segmentation results of the LBF model, the LGDF model, the LCV model, the LGIF model and our method, respectively.

In the sixth experiment, we quantitatively compare our method with the MICO [27] model and three well-known softwares, namely FSL ${ }^{1}$, SPM ${ }^{2}$ and FANTAS$\mathrm{M}^{3}$ in segmenting images from BrainWeb ${ }^{4}$. In order to compare with the MICO model more fairly, we use the same modality to generate images with intensity inhomogeneity in this experiment. First, the degree of intensity inhomogeneity is indicated by the range of values of the bias field in the interval $[1-\alpha, 1+\alpha]$ with $\alpha=0.1,0.2,0.3,0.4$ and 0.5 , respectively. Then, we generate six different bias fields with values in $[1-\alpha, 1+\alpha]$ for each given $\alpha$ and multiple them with the original image downloaded from BrainWeb to obtained six images with different intensity inhomogeneities. Finally, we add six different levels of noise to these images. As a result, there are 30 images with different degrees of intensity inhomogeneities and different levels of noise for each input image. In Fig 9, we

\footnotetext{
${ }^{1}$ http://www.fmrib.ox.ac.uk/fsl/

${ }^{2} \mathrm{http} / / / \mathrm{www}$.fil.ion.ucl.ac.uk/spm/software/

${ }^{3}$ http://www.mipav.cit.nih.gov/

${ }^{4}$ https://www.nitrc.org/projects/ibsr/
} 
first show the segmentation results of the five test methods in segmenting two of the 30 images for visual comparison, where the first row shows the segmentation results of image with the lowest degree of intensity inhomogeneity and the second row shows the segmentation results of image with the highest degree of intensity inhomogeneity. For quantitatively comparisons, we then give the box plot of the JS values for the GM and WM obtained from the five methods in Fig 10, which shows that our method have better performance than the other four method in terms of segmentation accuracy and robustness. Furthermore, by doing the experiment of our method three times under different initializations, we take the paired T-test to compare the segmentation results to illustrate the robustness of our method. The analysis results are shown in Table 5, which clearly illustrates that our method performs robustly under different initializations at the significance level 0.05 , because all the T-values are smaller than 2.042. Combining the comparison result shown in Fig 10, we can conclude that the significance of our method is due to the algorithm, not due to the initialization.

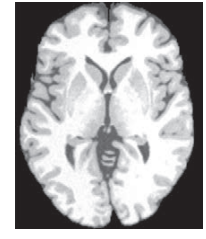

(a)

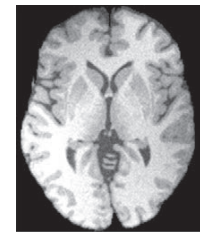

(g)

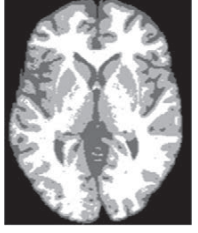

(b)

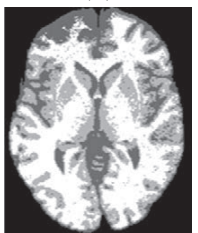

(h)

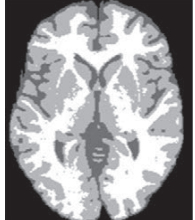

(c)

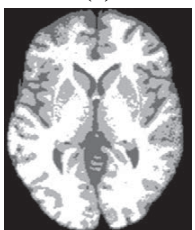

(i)

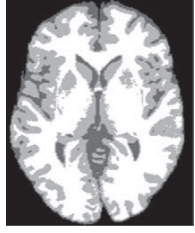

(d)

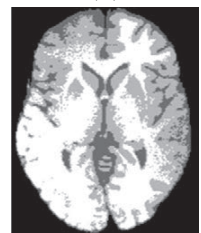

(j)

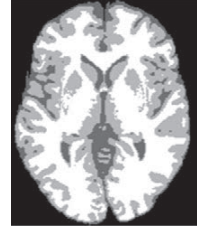

(e)

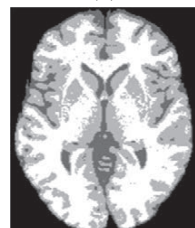

(k)

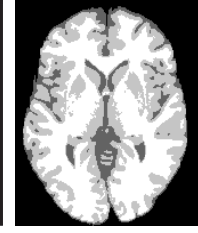

(f)

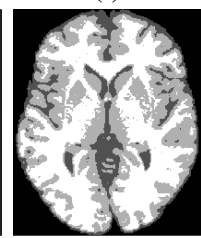

(l)

Figure 9: Comparison of our method with SPM, FSL, FANTASN and the MICO model in segmenting images with different intensity inhomogeneities. The input images are shown in the first column, and the segmentation results of SPM, FSL, FANTASN, the MICO model and our method are shown in the second column to the sixth column, respectively.

Table 5: Paried T-test of our method under three different initializations

\begin{tabular}{lllllll}
\hline Compared pairs & \multicolumn{2}{l}{ Initialization(1,2) } & \multicolumn{2}{l}{ Initialization(1,3) } & \multicolumn{2}{l}{ Initialization(2,3) } \\
\hline Tissue region & GM & WM & GM & WM & GM & WM \\
\hline T Value & 1.1373 & 1.4623 & 0.0977 & 0.0518 & 1.5162 & 1.1494 \\
\hline
\end{tabular}




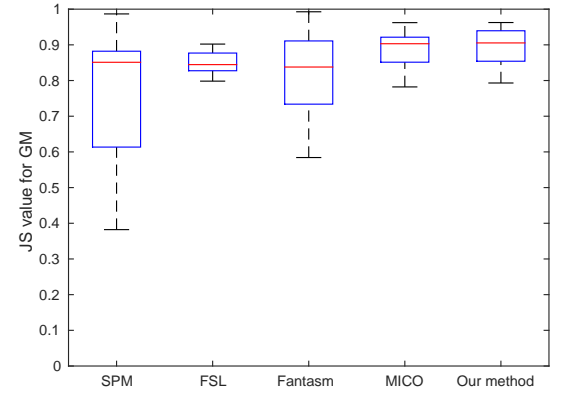

(a)

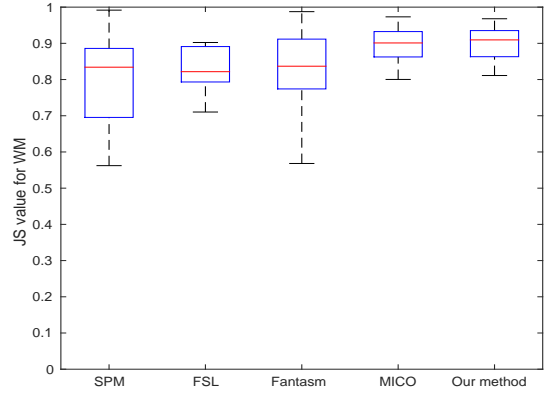

(b)

Figure 10: Accuracy comparison of our method with SPM, FSL, Fantasm and the MICO model using Jarcard similarity. (a) evaluation results for GM, (b) evaluation results for WM.

\section{Conclusion}

In this paper, we proposed an improved region-based active contour model in the level set formulation for medical image segmentation. In our method, the global energy term accelerates the evolution of the evolving curves when they are far away from the object boundaries, and the local energy term guides the evolving curves to stop on the precise object boundaries. The balance between the global energy term and the local energy term can be accommodated automatically by the introduced weighting function, which is based on the global and local intensity variance information. In addition, the a priori probability of a pixel belonging to the neighborhood partitions are modeled by using the size information of the local region at the two sides of the evolving curves. As a result, our model not only can segment medical images with intensity inhomogeneity, but also allows flexible initializations. Extensive results on both synthetic and real medical images show clear improvements on both efficiency and accuracy, as compared to the popular methods.

\section{Appendix A}

The regularization energy term defined in Eq. 177) consists of two energy terms, namely the distance penalty term $P(\phi)$ and the length penalty term $L(\phi)$, respectively. To understand the geometric meanings of the two energy terms, we give some analysis of how the two energy terms work for the final results. In calculus of variations, a standard method to minimize an energy functional $F(\phi)$ is to find a state solution of the gradient flow equation

$$
\frac{\partial \phi}{\partial t}=-\frac{\partial F}{\partial \phi}
$$


where $\partial F / \partial \phi$ is the Gateaux derivative of the energy functional $F(\phi)$.

The distance penalty term is designed to avoid the expensive re-initialization step by penalizing the distance between the level set function $\phi$ and the signed distance function $|\phi|=1$. By calculus of Eq. (15) with respect to $\phi$, the gradient flow of the distance penalty term can be written as follows:

$$
\frac{\partial \phi_{P}}{\partial t}=-\frac{\partial P(\phi)}{\partial \phi}=-\left(\Delta \phi-\operatorname{div}\left(\frac{\nabla \phi}{|\nabla \phi|}\right)\right)=-\operatorname{div}\left(\left(1-\frac{1}{|\nabla \phi|}\right) \nabla \phi\right)
$$

where $1-1 /|\nabla \phi|$ represents the diffusion rate of the distance penalty term. If $|\nabla \phi|>1$, the diffusion rate is positive and the effect of this term is forward diffusion, which decreases $|\nabla \phi|$. If $|\nabla \phi|<1$, the diffusion rate is negative and the effect of this term is backward diffusion, which increase $|\nabla \phi|$. As a result, the level set function can approach the signed distance function adaptively under the force of the distance penalty energy term.

The length penalty term is designed to regularize the zero level set by penalizing its length to derive a smooth contour during evolution. By calculus of Eq. (16) with respect to $\phi$, the gradient flow of the length penalty term can be written as follows:

$$
\frac{\partial \phi_{L}}{\partial t}=-\frac{\partial L(\phi)}{\partial \phi}=\delta(\phi) \operatorname{div}\left(\frac{\nabla \phi}{|\nabla \phi|}\right)=\delta(\phi) \operatorname{div}\left(\frac{1}{|\nabla \phi|} \nabla \phi\right)
$$

where $1 /|\nabla \phi|$ represents the diffusion rate of the length penalty term. If $|\nabla \phi|>1$, the diffusion rate is smaller than 1, which will slow down the speed to decrease $|\nabla \phi|$. If $|\nabla \phi|<1$, the diffusion rate is bigger than 1, which will slow down the speed to increase $|\nabla \phi|$. As a result, the effect of the length penalty is to make the contour evolution more stable, which will ensure the contour smooth during the evolution.

\section{References}

[1] Y. Chen, J. Zhang, J. Yang, An anisotropic images segmentation and bias correction method, Magnetic resonance imaging 30 (1) (2012) 85-95.

[2] W. C. Wong, A. C. Chung, Bayesian image segmentation using local iso-intensity structural orientation, Image Processing, IEEE Transactions on 14 (10) (2005) 15121523.

[3] S. P. Awate, T. Tasdizen, N. Foster, R. T. Whitaker, Adaptive markov modeling for mutual-information-based, unsupervised mri brain-tissue classification, Medical Image Analysis 10 (5) (2006) 726-739. 
[4] L. Wang, L. He, A. Mishra, C. Li, Active contours driven by local gaussian distribution fitting energy, Signal Processing 89 (12) (2009) 2435-2447.

[5] L. Dai, J. Ding, J. Yang, Inhomogeneity-embedded active contour for natural image segmentation, Pattern Recognition 48 (8) (2015) 2513-2529.

[6] M. Styner, C. Brechbuhler, G. Szckely, G. Gerig, Parametric estimate of intensity inhomogeneities applied to mri, Medical Imaging, IEEE Transactions on 19 (3) (2000) $153-165$.

[7] E. B. Lewis, N. C. Fox, Correction of differential intensity inhomogeneity in longitudinal mr images, Neuroimage 23 (1) (2004) 75-83.

[8] C. Li, R. Huang, Z. Ding, C. Gatenby, D. Metaxas, J. Gore, A variational level set approach to segmentation and bias correction of images with intensity inhomogeneity, in: Medical Image Computing and Computer-Assisted Intervention-MICCAI 2008, Springer, 2008, pp. 1083-1091.

[9] C. Li, R. Huang, Z. Ding, J. Gatenby, D. N. Metaxas, J. C. Gore, A level set method for image segmentation in the presence of intensity inhomogeneities with application to mri, Image Processing, IEEE Transactions on 20 (7) (2011) 2007-2016.

[10] T. Zhan, J. Zhang, L. Xiao, Y. Chen, Z. Wei, An improved variational level set method for $\mathrm{mr}$ image segmentation and bias field correction, Magnetic resonance imaging 31 (3) (2013) 439-447.

[11] Y. Chen, J. Zhang, J. Macione, An improved level set method for brain mr images segmentation and bias correction, Computerized Medical Imaging and Graphics 33 (7) (2009) 510-519.

[12] Y. Chen, J. Zhang, A. Mishra, J. Yang, Image segmentation and bias correction via an improved level set method, Neurocomputing 74 (17) (2011) 3520-3530.

[13] V. Caselles, R. Kimmel, G. Sapiro, Geodesic active contours, in: Computer Vision, IEEE International Conference on, IEEE Computer Society, 1995, pp. 694-694.

[14] G. Zhu, S. Zhang, Q. Zeng, C. Wang, Boundary-based image segmentation using binary level set method, Optical Engineering 46 (5) (2007) 050501-050501.

[15] C. Li, C. Xu, C. Gui, M. D. Fox, Level set evolution without re-initialization: a new variational formulation, in: Computer Vision and Pattern Recognition, 2005. CVPR 2005. IEEE Computer Society Conference on, Vol. 1, IEEE, 2005, pp. 430-436.

[16] N. Paragios, R. Deriche, Geodesic active contours and level sets for the detection and tracking of moving objects, Pattern Analysis and Machine Intelligence, IEEE Transactions on 22 (3) (2000) 266-280. 
[17] N. Paragios, R. Deriche, Geodesic active regions and level set methods for supervised texture segmentation, International Journal of Computer Vision 46 (3) (2002) 223247.

[18] A. Vasilevskiy, K. Siddiqi, Flux maximizing geometric flows, Pattern Analysis and Machine Intelligence, IEEE Transactions on 24 (12) (2002) 1565-1578.

[19] T. F. Chan, L. A. Vese, Active contours without edges, Image processing, IEEE transactions on 10 (2) (2001) 266-277.

[20] D. Cremers, M. Rousson, R. Deriche, A review of statistical approaches to level set segmentation: integrating color, texture, motion and shape, International journal of computer vision 72 (2) (2007) 195-215.

[21] A. Tsai, A. Yezzi Jr, A. S. Willsky, Curve evolution implementation of the mumfordshah functional for image segmentation, denoising, interpolation, and magnification, Image Processing, IEEE Transactions on 10 (8) (2001) 1169-1186.

[22] L. A. Vese, T. F. Chan, A multiphase level set framework for image segmentation using the mumford and shah model, International journal of computer vision 50 (3) (2002) 271-293.

[23] C. Li, C.-Y. Kao, J. C. Gore, Z. Ding, Minimization of region-scalable fitting energy for image segmentation, Image Processing, IEEE Transactions on 17 (10) (2008) 1940-1949.

[24] C. Li, C.-Y. Kao, J. C. Gore, Z. Ding, Implicit active contours driven by local binary fitting energy, in: Computer Vision and Pattern Recognition, 2007. CVPR'07. IEEE Conference on, IEEE, 2007, pp. 1-7.

[25] B. Rosenhahn, T. Brox, J. Weickert, Three-dimensional shape knowledge for join$\mathrm{t}$ image segmentation and pose tracking, International Journal of Computer Vision 73 (3) (2007) 243-262.

[26] L. Wang, C. Pan, Robust level set image segmentation via a local correntropy-based k-means clustering, Pattern Recognition 47 (5) (2014) 1917-1925.

[27] C. Li, J. C. Gore, C. Davatzikos, Multiplicative intrinsic component optimization (mico) for mri bias field estimation and tissue segmentation, Magnetic resonance imaging 32 (7) (2014) 913-923. 
Sanping Zhou received the M.E. degree from Northwestern Polytechnical University, Xi'an, China, in 2015. He is currently pursuing the Ph.D. degree in Institute of Artificial Intelligence and Robotics at Xi' an Jiaotong University. His research interests include medical image segmentation, deep learning, person re-identification, and visual tracking.

Jinjun Wang received the B.E. and M.E. degrees from the Huazhong University of Science and Technology, China, in 2000 and 2003, respectively. He received the Ph.D. degree from Nanyang Technological University, Singapore, in 2006. From 2006 to 2009, he was with NEC Laboratories America, Inc., as a Research Scientist, and Epson Research and Development, Inc., as a Senior Research Scientist, from 2010 to 2013. He is currently a Professor with Xi'an Jiaotong University. His research interests include pattern classification, image/video enhancement and editing, content-based image/video annotation and retrieval, semantic event detection, etc.

Shun Zhang received the B.S. degree in Electronic Engineering from Xi'an Jiaotong University, Xi'an, China, in 2009. He is currently pursuing the Ph.D. degree in Institute of Artificial Intelligence and Robotics at Xi' an Jiaotong University. His research interests include Machine Learning, Computer Vision and Human-Computer Interaction, with a focus on visual tracking, object detection, image classification, feature extraction and sparse representation.

Yudong Liang received the B.S. degree in Electronic Engineering from Xi'an Jiaotong University, Xi'an, China, in 2010. He is currently pursuing the Ph.D. degree in Institute of Artificial Intelligence and Robotics at Xi'an Jiaotong University. His research interests include Machine Learning and Computer Vision, with a focus on image superresolution, image quality assessment and deep learning.

Yihong Gong received the B.S., M.S., and Ph.D. degrees in electrical engineering from The University of Tokyo, Japan, in 1987, 1989, and 1992, respectively. In 1992, he joined Nanyang Technological University, Singapore, as an Assistant Professor with the School of Electrical and Electronic Engineering. From 1996 to 1998, he was a Project Scientist with the Robotics Institute, Carnegie Mellon University, USA. Since 1999, he has been with the Silicon Valley branch, NEC Labs America, as a Group Leader, the Department Head, and the Branch Manager. In 2012, he joined Xi'an Jiaotong University, China, as a Distinguished Professor. His research interests include image and video analysis, multimedia database systems, and machine learning. 
${ }^{*}$ Photo of the author(s)

Click here to download high resolution image

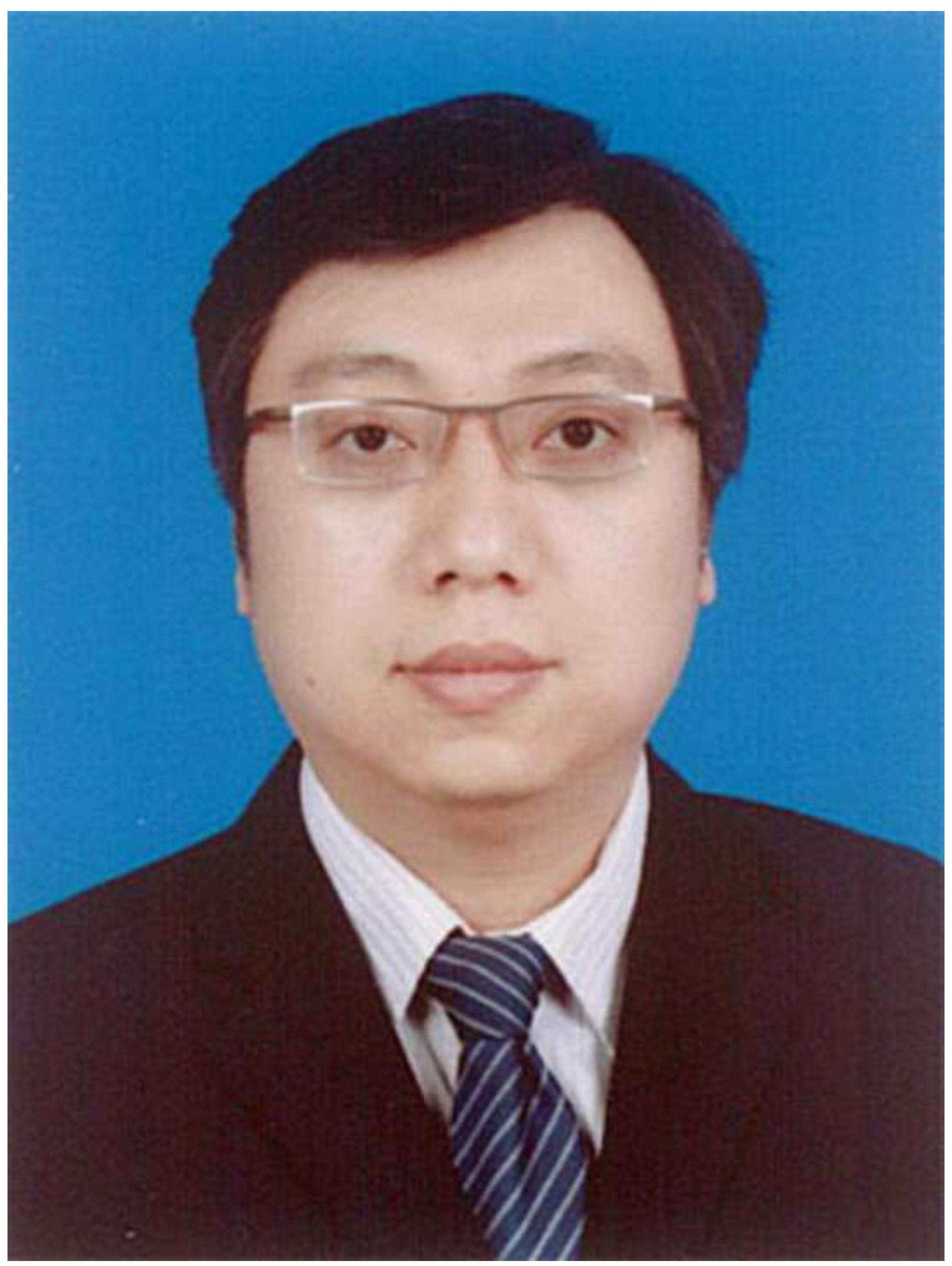


${ }^{*}$ Photo of the author(s)

Click here to download high resolution image

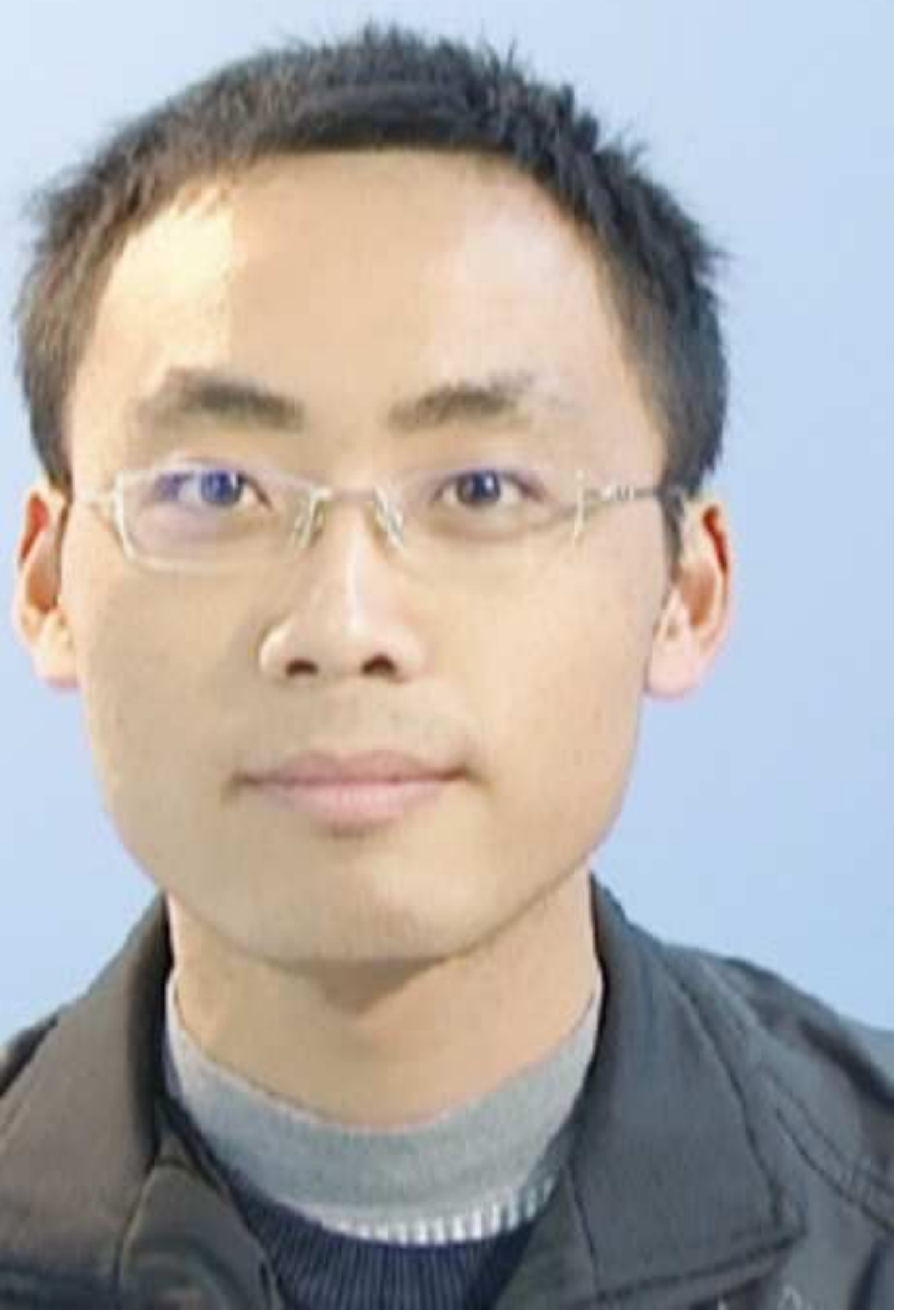


${ }^{*}$ Photo of the author(s)

Click here to download high resolution image

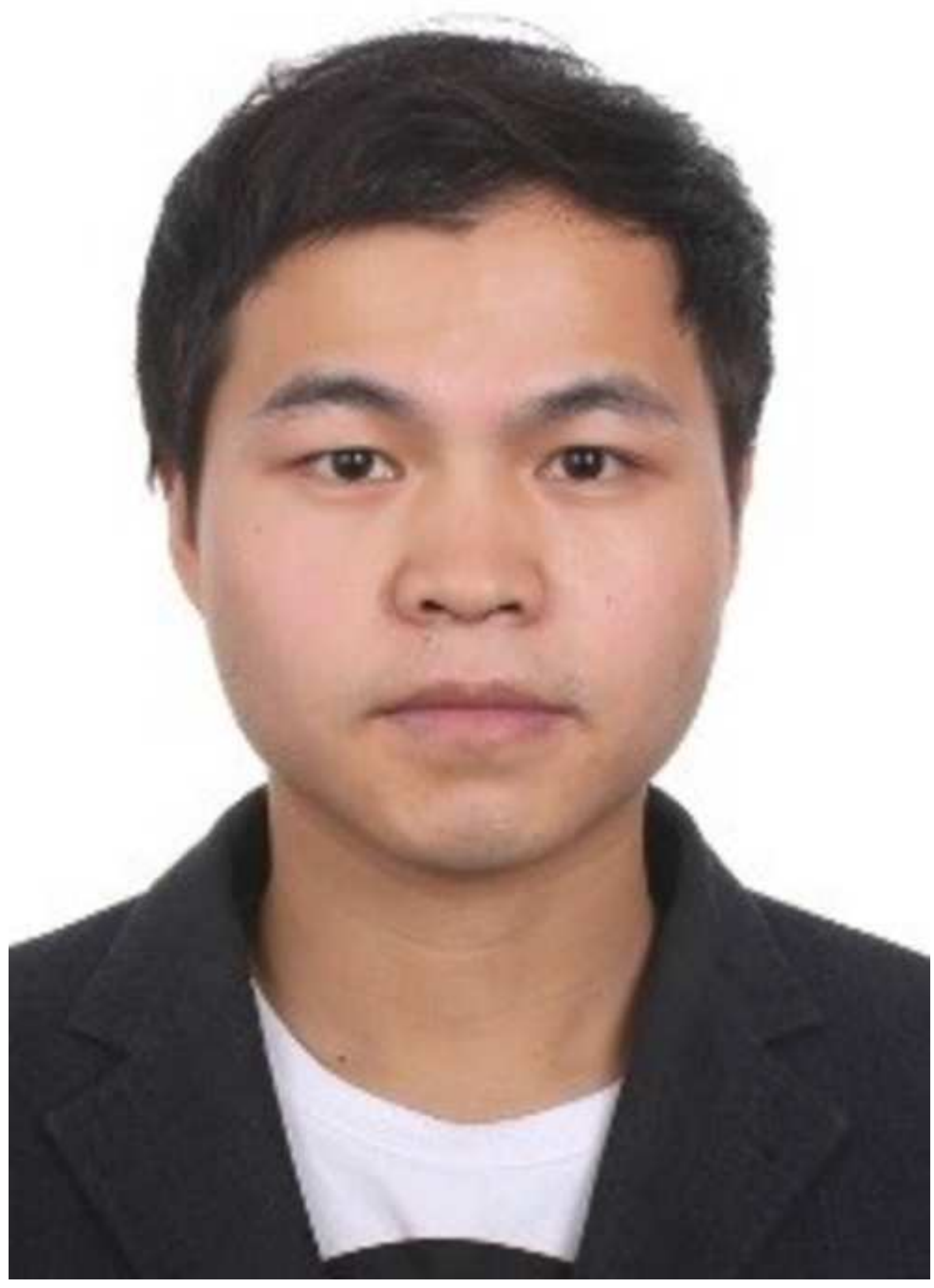




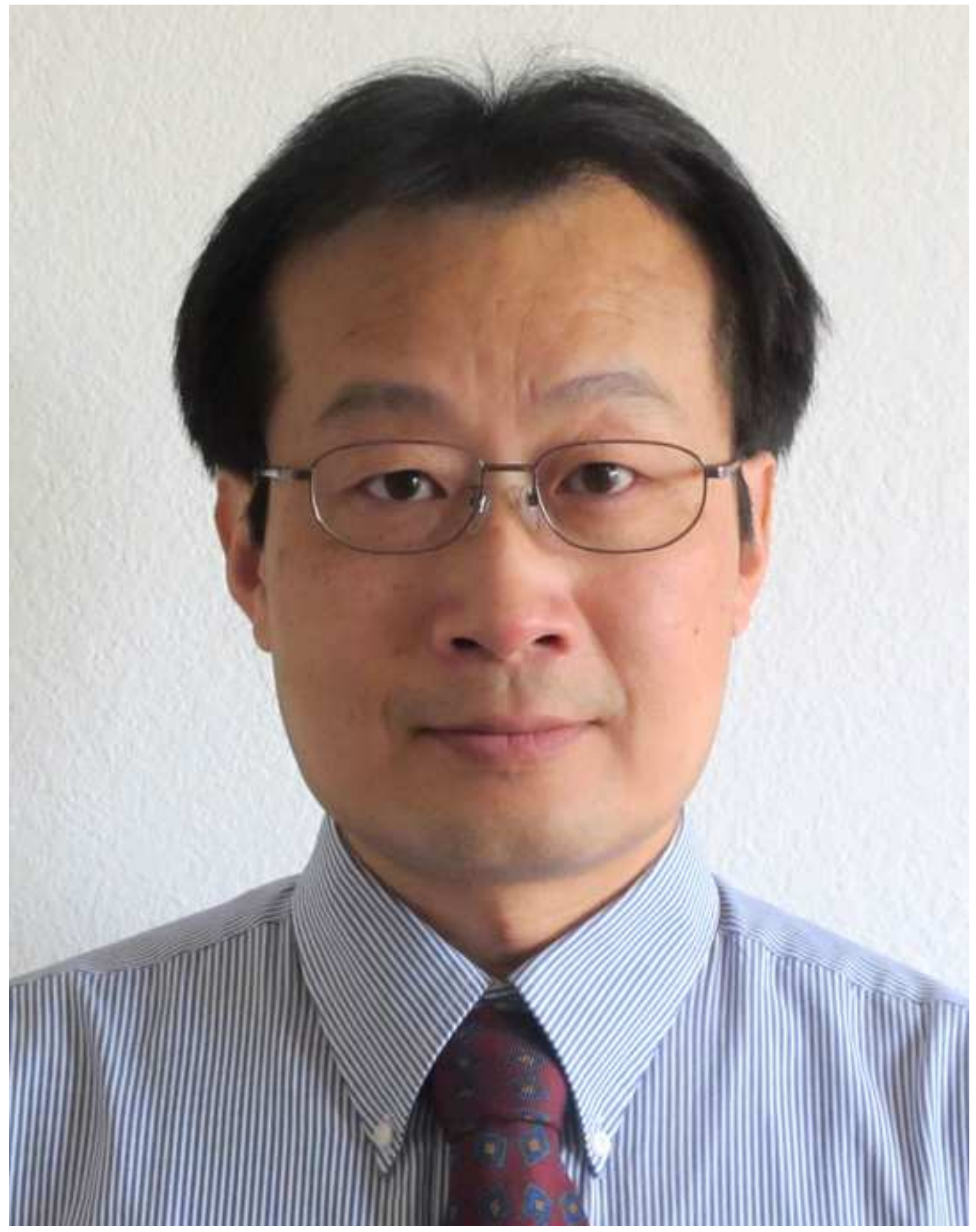


${ }^{*}$ Photo of the author(s)

Click here to download high resolution image

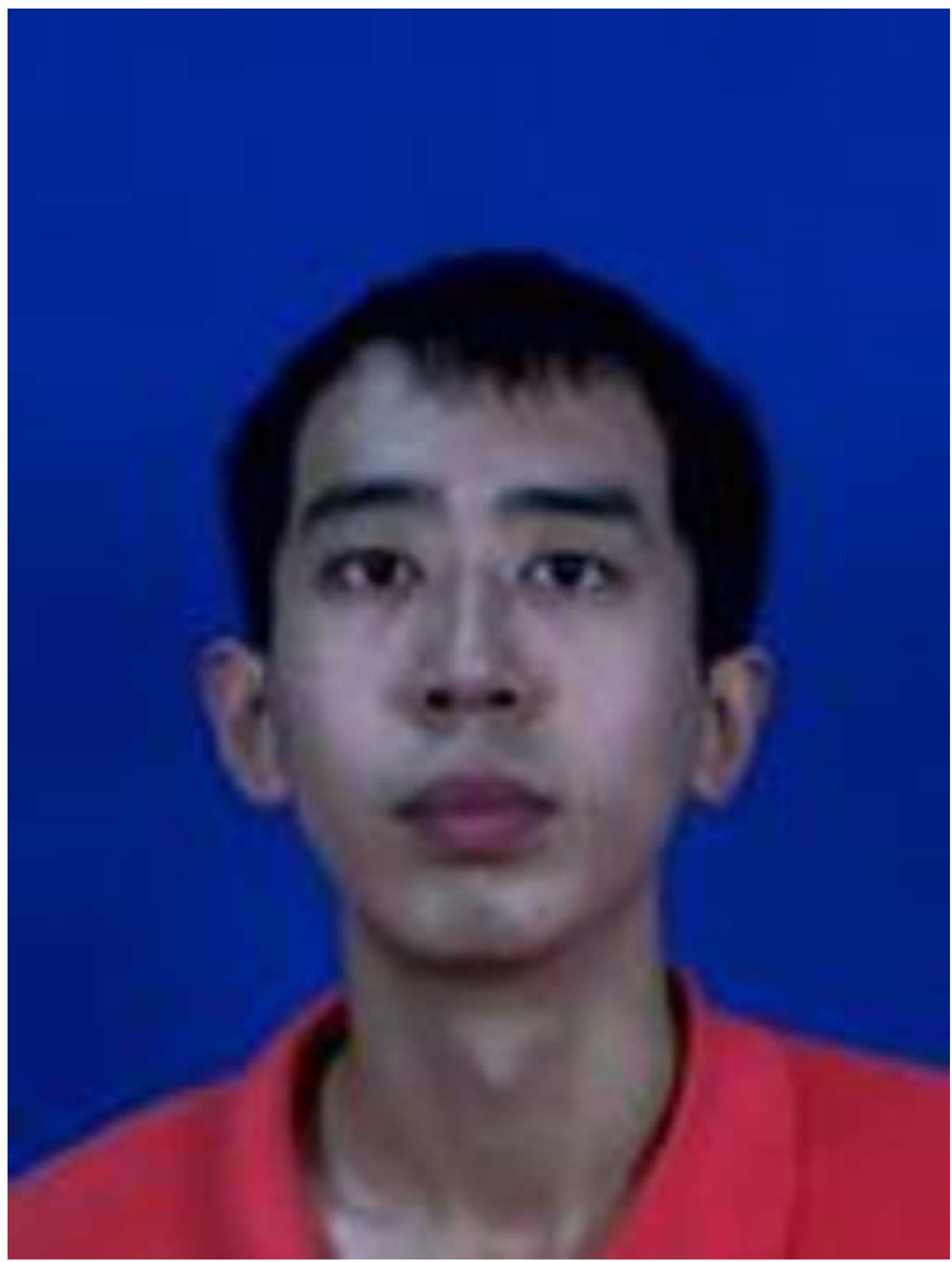

